\title{
Broadcast Network Coverage with Multicell Cooperation
}

\author{
Hongxiang Li, ${ }^{1}$ Samee Ullah Khan, ${ }^{1}$ and Hui Liu' ${ }^{2}$ \\ ${ }^{1}$ Electrical and Computer Engineering, North Dakota State University, Fargo, ND 58102, USA \\ ${ }^{2}$ Electrical Engineering, University of Washington, Seattle, WA 98195, USA
}

Correspondence should be addressed to Hongxiang Li, hongxiang.li@ndsu.edu

Received 17 August 2009; Accepted 22 October 2009

Academic Editor: Lingjia Liu

Copyright (C) 2010 Hongxiang Li et al. This is an open access article distributed under the Creative Commons Attribution License, which permits unrestricted use, distribution, and reproduction in any medium, provided the original work is properly cited.

\begin{abstract}
Multicell cooperation has been identified as one of the underlying principles for future wireless communication systems. This paper studies the benefits of multicell cooperation in broadcast TV network from an information theoretical perspective. We define outage capacity as the figure of merit and derive the broadcast coverage area to evaluate such system. Specifically, we calculate the broadcast coverage area with given common information rate and outage probabilities when multiple base stations collaboratively transmit the broadcast signals. For the general MIMO case where receivers have multiple antennas, we provide simulation results to illustrate the expanded coverage area. In all cases, our results show that the coverage of a TV broadcast network can be significantly improved by multicell cooperation.
\end{abstract}

\section{Introduction}

The wireless industry is experiencing an unprecedented increase in demand for better multimedia broadcasting systems. The growing diffusion of new services, like HDTV broadcasting and mobile television, emphasize the need of advanced transmission techniques that can fundamentally increase the system performance over the conventional broadcast network. Cooperative transmission is a key technology that has been identified to fulfill this demand.

Most of the studies on cooperative transmission so far focus on reducing intercell interference and increasing system capacity in two-way (uplink/downlike) cellular systems, where cooperation can be employed at either the base station (BS) or mobile station (MS). Multicell cooperation (MCC), sometimes also referred to as multicell processing or distributed antenna system, prescribes joint encoding/decoding of the signals transmitted/received at the BSs through the exploitation of the high-capacity backbone connecting the BSs. A survey on MCC can be found in [1-4]. Assume all the BSs in the network are connected to a central processor via links of unlimited capacity, the set of BSs effectively acts as a geographically distributed multiantenna system. Under this perfect MCC assumption, [5] investigated multicell downlink channel capacity with single-class network where the users are clustered at the cell edges. The analytical performance expression for dirty paper coding (DPC), cophasing, zero forcing (ZF), and MMSE precoders are derived in this paper. Recently [6] studied the capacity of a cellular system with a large number of BSs and MSs. The evaluation is based on an accurate modeling of the multicell deployment, different level of cooperation among BSs, and practical per-antenna transmit power constraint of a BS transmit antenna. The performance upper and lower bounds are also derive for different cooperation scenarios. On the other hand, the impact of limited-capacity backhaul on MCC (i.e., partial cooperation) has also been studied in the literature [7-14]. In [7], the authors studied achievable rates of a multicell network under the assumption that BS and MS cooperation are enabled by error-free but limitedcapacity interbase station and interuser links, respectively. The analytical treatment enhances the insight into the potential and the limitations imposed by capacity constraints on the performance gains provided by cooperative techniques. Some recent results on intercell interference coordination through limited feedback can also be found in [14].

While there are many existing work showing the benefits of MCC in cellular networks, few studies can be found in the literature on cooperative broadcast network. Compared 
with the cellular (i.e., voice and data) network, there are some fundamental differences in broadcast (i.e, TV) network.

(i) Communication is only one-way from base station to subscriber stations (SS); there is no reverse link available. Therefore, the channel state information (CSI) is not available at the transmitter. In cellular network, reverse link is available and the BS can have perfect or partial CSI.

(ii) BS sends the same common information to all the subscribers. In cellular network, BS sends different individual information to each subscriber.

(iii) SSs (TV receivers) can only receive but not transmit signals. Therefore, there is no possibility of cooperation (information exchange) among SSs. In cellular network, MSs (smart phones, laptops) can transmit signals and thus cooperation is possible among MSs.

Unlike the two-way cellular network, the concern of broadcast TV network is usually not the throughput. Rather, the design objective of such network is to maximize the broadcast coverage, with a predetermined transmission rate. It has been expected that MCC can extend the broadcast coverage area, forming an extended ellipse coverage beyond the superposition of individual cells [15]. However, to the best knowledge of the author of this article, there are no quantitative studies in the literature on how much the broadcast coverage can be extended by MCC. In this paper, we consider full MCC in broadcast network with cooperation among some small number of neighboring base stations. Specifically, we define the figure-of-merit of MCC in broadcast from an information theoretical perspective. Then we quantify the extended coverage areas under different MCC scenarios and derive the coverage gains over single-cell independent transmission. We also investigate the broadcast coverage with optimal base station separation. This paper is an extension of our earlier work [16], where we calculated the broadcast coverage in a broadcast and unicast hybrid network with three collaborating cells.

The paper is organized as the following. In Section 2, we define the performance metric and present the broadcast channel model. We then analyze and compute the broadcast coverage with MCC for single/multiple receiving antenna(s) in Section 3. Finally, a conclusion is drawn in Section 4.

To facilitate our discussion, we use the following notation conventions throughout this paper:

$$
\begin{aligned}
& N_{r}: \text { the number of antennas at each receiver } \\
& N_{t}: \text { the number of collaborating base stations } \\
& \mathbf{H}_{k}: N_{r} \times N_{t} \text { (channel matrix associated with user } k \text { ) } \\
& \text { Q: input spatial covariance matrix } \\
& P \text { : the total transmit power } \\
& B \text { : the total channel bandwidth } \\
& r_{o}: \text { common information broadcast rate } \\
& q_{o}: \text { outage Probability } \\
& N_{0}: \text { white noise variance }\left(10^{-9} \mathrm{w} / \mathrm{Hz}\right)
\end{aligned}
$$

\section{System Model}

In wireless community, the term broadcast has been used for both TV broadcast and cellular downlink system. To avoid ambiguity, we define broadcast network as the delivery of common information from the base station or TV tower to silent users in multicast applications. As we discussed in Section 1, there is no reverse link and thus CSI is not available at the transmitter.

In order to quantify the performance of a broadcast system, we must first define the performance metric. In information theory, there are two channel capacity definitions that are relevant to the system design for a broadcast channel with an uninformed transmitter: the ergodic capacity (also called the Shannon capacity) and the outage capacity [17]. The ergodic capacity defines the maximum data rate that can be sent to the receiver with asymptotically small error probability through all the fading states. In most cases, TV receivers at fixed locations experience slow fading and the choose of ergodic capacity will incur intolerant decoding delays. On the other hand, the outage capacity defines the maximum data rate that can be transmitted with certain outage probability that the received data can not be decoded with negligible error probability. If the received SNR is above the threshold corresponding to the outage probability, the transmitted data can be decoded with negligible probability of error; otherwise, the transmission is in outage. By allowing some outage, the broadcast receiver can decode the message during each fading state and thus meet the delay requirement. In this paper, we use the more practical outage capacity as the figure of merit for evaluating the broadcast network.

In general, since broadcast receivers are geographically distributed over a large area, they decode the received signals with different outage probabilities depending on the fading statistics. Within a given coverage area $A$, We define the outage probability $q\left(W_{A}\right)$ as the outage probability (package loss rate) associated with the worst broadcast receiver $W$ in this area. Note that the worst receiver $W$ always experiences the highest outage in $A$. Literally, there are three interchangeable ways to optimize the broadcast performance.

(1) Given a broadcast rate $r_{o}$ and an outage probability $q_{o}$, maximize the coverage area $A$ such that $q\left(W_{A}\right) \leq$ $q_{0}$.

(2) Given a coverage area $A$ and an outage probability $q_{o}$, maximize the common information rate $r_{o}$ such that $q\left(W_{A}\right) \leq q_{o}$.

(3) Given the broadcast rate $r_{o}$ and coverage area $A$, minimize the worst user outage probability $q\left(W_{A}\right)$.

Note that the above three problems are equivalent in optimizing the common information transmission from BS to receiver $W_{A}$. In other words, we only need to focus on the performance of the worst receiver in the covered area. In practical TV broadcast networks, $r_{o}$ and $q_{o}$ are usually prefixed and thus our objective is to maximize broadcast coverage area with given quality of service (outage) requirement. 
In conventional TV broadcasting, the terrestrial television stations are usually high power tall towers that cover a large area and the TV receivers are at fixed locations. With the increasing demand of mobile TV, many wireless carriers are offering TV services over their cellular base stations to mobile subscribers with smart phones or PDAs. In U.S., wireless carriers (Verizon, AT\&T mobility and etc.) are using MediaFlo (forward link only) technology to deliver mobile TV service. In this paper, we are not limited to a particular broadcast network or technique, rather, we study the broadcast coverage from an information theory point of view. To ease our discussion, we simply use the term "base station" to refer any broadcast transmitter (radio mast, tower or cellular base station). In traditional broadcast network, base stations are operated independently. In this paper, we investigate the scenario where multiple base stations can collaborate with each other to transmit the common information.

To calculate the broadcast coverage, we have to assume the channel fading statistics. The same channel model in [16] is used. For free space propagation loss, Hata model is the most common model for signal prediction in large urban macro-cells. This model is applicable over distances of $1-100 \mathrm{~km}$ and frequency ranges of $150-1500 \mathrm{MHz}$. The standard formula for empirical path loss in urban areas under the Hata model is

$$
\begin{aligned}
P_{L, \text { urban }}(d) \mathrm{dB}= & 26.16 \log _{10}\left(f_{c}\right)-13.82 \log _{10}\left(h_{t}\right) \\
& -a\left(h_{r}\right)+\left(44.9-6.55 \log _{10}\left(h_{t}\right)\right) \log _{10}(d) \\
& +69.55,
\end{aligned}
$$

where $f_{c}$ is the carrier frequency, $h_{t} / h_{r}$ is the transmitter/receiver antenna height and $d$ is the distance between transmitter and receiver. For larger cities at frequencies $f_{c}>$ $300 \mathrm{MHz}$, the correction factor $a\left(h_{r}\right)$ is given by

$$
a\left(h_{r}\right)=3.2\left(\log _{10}\left(11.75 h_{r}\right)\right)^{2}-4.97 \mathrm{~dB} .
$$

For small scale fading, we assume Rayleigh flat fading, that is, the envelope of the complex channel gain for each spatial channel has the following distribution:

$$
f(|h|)=\frac{|h|}{\sigma^{2}} \exp \left(-\frac{|h|^{2}}{\sigma^{2}}\right)
$$

where $E\left[|h|^{2}\right]=2 \sigma^{2}$ is determined by path loss in (1).

Note that in a real urban environment with many highrise buildings, the actual channel statistics is much more complicated. A combined path loss and shadowing model is needed to calculate an outage of a particular receiver. However, this fading statistics will highly depends on the actual urban terrain and structure and varies from city to city. Assume a flat open area, our simplified channel model can give enough insights on the benefit of MCC. We also assume slow flat fading throughout the paper.

\section{Broadcast Coverage}

In a single cell broadcast network with a fixed broadcast rate $r_{o}$, the information theoretical outage probability associated with receiver $k$ is given by [17]

$$
q_{o}(k)=\operatorname{Prob}\left[h_{k}: B \log _{2}\left(1+\frac{P\left|h_{k}\right|^{2}}{N_{0} B}\right)<r_{o}\right] .
$$

In continuous frequency-selective case, the outage probability $q_{o}(k)$ becomes

$$
\begin{aligned}
& \operatorname{Prob}\left[h_{k}: \int_{0}^{B} \log _{2}\left(1+\frac{P(f)\left|h_{k}(f)\right|^{2}}{N_{0}}\right) d f<r_{o}\right] \\
& \text { s.t. } \int_{0}^{B} P(f) d f=P .
\end{aligned}
$$

In multicarrier/OFDM system where the channel is block frequency-selective, (5) becomes

$$
\begin{aligned}
& \operatorname{Prob}\left[h_{k}: \sum_{i=1}^{N} B_{n} \log _{2}\left(1+\frac{P(i)\left|h_{k}(i)\right|^{2}}{N_{0} B_{n}}\right)<r_{o}\right] \\
& \text { s.t. } \sum_{i=1}^{N} P(i)=P
\end{aligned}
$$

Obviously, the coverage area $A$ determined by Equation (1)-(4) is a circle for any given outage requirement. Without loss of generality, we choose $r_{o}=515 \mathrm{Kbps}$ and $P=1$ watt such that the radius $d_{0}=1 \mathrm{~km}$ is the benchmark distance with $q_{o}\left(d_{0}\right)=5 \%$. Figure 1 shows the broadcast coverage area with different outage probabilities, where the solid black curve ( $5 \%$ outage) is our benchmark. We see that the coverage increases with $q_{o}$ as expected.

Next, we will discuss the coverage expansion by multicell cooperation.

3.1. MCC with Single Receiver Antenna $\left(N_{r}=1\right)$. We assume multiple base stations are wire connected through highcapacity backbone network and thus can fully collaborate with each other. In this case, each base station can be viewed as a transmitting antenna of a distributed multiple input single output (MISO) system. Also, because the distances among base stations are on the order of kilometers, spatial channels from different base stations are considered mutually independent. Let $\overrightarrow{\mathbf{x}}=\left[x(1), x(2), \ldots, x\left(N_{t}\right)\right]^{H}$ be the transmitted input vector, the outage probability associated with receiver $k$ is given by [17]

$$
\begin{gathered}
q_{o}=\operatorname{Prob}\left[\overrightarrow{\mathbf{h}_{k}}: B \log _{2} \operatorname{det}\left(\mathbf{I}+\frac{\overrightarrow{\mathbf{h}_{k}} \mathbf{Q h}_{k}^{\vec{H}}}{N_{0} B}\right)<r_{o}\right] \\
\text { s.t. } \mathbf{E}\left[x(i) x(i)^{*}\right]=\frac{P}{N_{t}}, \quad 1 \leq i \leq N_{t},
\end{gathered}
$$

where $\overrightarrow{\mathbf{h}_{k}}=\left[h_{k}(1), h_{k}(2), \ldots, h_{k}\left(N_{t}\right)\right]$ is the channel vector and $\mathbf{Q}=\mathbf{E}\left[\overrightarrow{\mathbf{x}}^{H}\right]$ is the spatial input covariance matrix. 


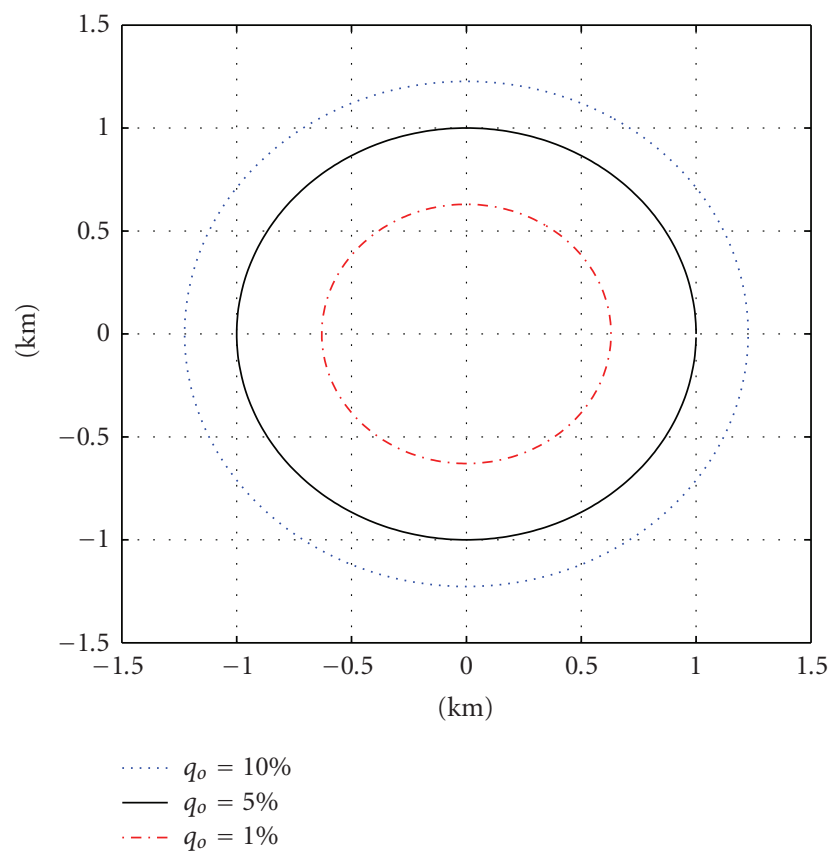

FIGURE 1: Single cell broadcast coverage.

Remark 1. In practice, each base station is subject to its own power constraint, which is dictated by the amplifier of the transmitting antenna. In this paper, we adopt this individual BS power constraint. However, for fair comparison of broadcast coverage between single cell and multicell cases, we put a total power cap in multicell transmission, that is, the maximum sum power over all base stations is $P$.

Because of the nature of the broadcast network, the BSs do not have the channel and location information of the receivers. Therefore, the transmitter cannot optimize its input covariance structure across antennas for every receiving point simultaneously. Assume the zero-mean spatially white (ZMSW) channel model, the optimal power allocation strategy is to allocate equal power to each base station [18], resulting $\mathbf{Q}$ equal to the scaled identity matrix: $\mathbf{Q}=\left(\mathbf{P} / \mathbf{N}_{\mathbf{t}}\right) \mathbf{I}$. Equation (7) thus becomes

$$
q_{o}=\operatorname{Prob}\left[\overrightarrow{\mathbf{h}_{k}}: B \log _{2} \operatorname{det}\left(\mathbf{I}+\frac{P}{N_{t}} \frac{\left\|\overrightarrow{\mathbf{h}_{k}}\right\|^{2}}{N_{0} B}\right)<r_{o}\right] .
$$

Note that equal power distribution over BSs does not mean all the BSs transmit the same signal. With channel unknown at transmitter, the Alamouti Scheme is a technique to achieve the $N_{t}$ fold diversity gain [17]. From (8), we can see that, given $r_{o}$ and $q_{o}$, the coverage area depends solely on the distribution of $\left\|\overrightarrow{\mathbf{h}}_{k}\right\|^{2}$. Since the average path loss is simply a function of the distance between base station and a broadcast receiver, we know the worst receiver $W_{A}$ is always on the edge of area $A$. Therefore, the coverage area can be computed by locating edge users in all directions. To do so, we first need to derive the distribution of $\left\|\overrightarrow{\mathbf{h}_{k}}\right\|^{2} / N_{t}$. In practice, it is unrealistic and unnecessary to do multicell cooperation for a large number of cells $[6,7]$. Here we study two practical scenarios where $N_{t}=2$ and $N_{t}=3$.

Let $Y=\|\overrightarrow{\mathbf{h}}\|^{2} / N_{t}$ and $X_{i}=|h(i)|^{2} / N_{t}$. Note that $X_{i}$ and $X_{j}$ are i.i.d $(i \neq j)$ random variables with chi-square distribution, that is, the distribution of $X_{i}$ is given by

$$
\begin{aligned}
& \text { PDF }: f(x)=\frac{1}{2 \sigma^{2}} \exp \left(-\frac{x}{2 \sigma^{2}}\right) \\
& \mathrm{CDF}: F(x)=1-\exp \left(-\frac{x}{2 \sigma^{2}}\right)
\end{aligned}
$$

For the case $N_{t}=2$, the cdf of $Y$ can be obtained by convoluting (9) as

$$
\begin{aligned}
F_{Y}(y)= & f_{X_{1}}(y) * F_{X_{2}}(y) \\
= & \left\{\begin{array}{l}
\text { if } \sigma_{1} \neq \sigma_{2} \\
1+\frac{1}{\sigma_{2}^{2}-\sigma_{1}^{2}}\left[\sigma_{1}^{2} \exp \left(-\frac{y}{2 \sigma_{1}^{2}}\right)-\sigma_{2}^{2} \exp \left(-\frac{y}{2 \sigma_{2}^{2}}\right)\right] \\
\text { if } \sigma_{1}=\sigma_{2} \\
1-\exp \left(-\frac{y}{2 \sigma_{1}^{2}}\right)-\frac{y}{2 \sigma_{1}^{2}} \exp \left(-\frac{y}{2 \sigma_{1}^{2}}\right),
\end{array}\right.
\end{aligned}
$$

where $\sigma_{i}$ is determined by the pass loss from base station $i$ to the receiver.

In the general case with arbitrary $N_{t}$, the PDF of $Y$ can be obtained as

$$
f_{Y}(y)=\left\{\begin{array}{l}
\text { if } \sigma_{1} \neq \sigma_{2} \neq \cdots \neq \sigma_{N} \\
\sum_{i=1}^{N} \frac{\sigma_{i}^{2 N-4}}{\prod_{j=1 j \neq i}^{N}\left(\sigma_{i}^{2}-\sigma_{j}^{2}\right)} \exp \left(-\frac{y}{\sigma_{i}^{2}}\right) \\
\text { if } \sigma_{1}=\sigma_{2}=\cdots=\sigma_{N} \\
\frac{y^{N-1}}{(N-1) ! \sigma^{2 N}} \exp \left(-\frac{y}{\sigma_{i}^{2}}\right) .
\end{array}\right.
$$

Equations (10) and (11) can be used to determine the multicell broadcast coverage area. For example, Figure 2 shows the two cell collaborating case, where $D$ is the cell separation distance and $(\rho, \theta)$ is the polar coordinates of the receiver. Note that for path loss, $\sigma_{i}$ is simply a function of the distance between cell $i$ and the receiver. For each direction $(0 \leq \theta \leq 360)$ from the origin, we calculate a radius $\rho(\theta)$ such that the distribution of $\|\overrightarrow{\mathbf{h}}\|^{2} / N_{t}$ at $(\rho, \theta)$ satisfies (8). By locating the coverage edge in all directions, the maximum coverage area can be numerically computed.

Figures 3-5 show some numerical results of multicell broadcast coverage. For $N_{t}=2$, Figure 3 plots the extended coverage with two base stations located at $\left(d_{0} / 2,0\right)$ and $\left(-d_{0} / 2,0\right)$. Compared to single cell transmission shown in Figure 1, the coverage gains with two collaborating cells are $3.5399,2.0922$ and 3.4522 for outage probability $1 \%, 5 \%$ and $10 \%$ respectively, where we define the coverage gain as the ratio of MCC coverage over single cell coverage. 


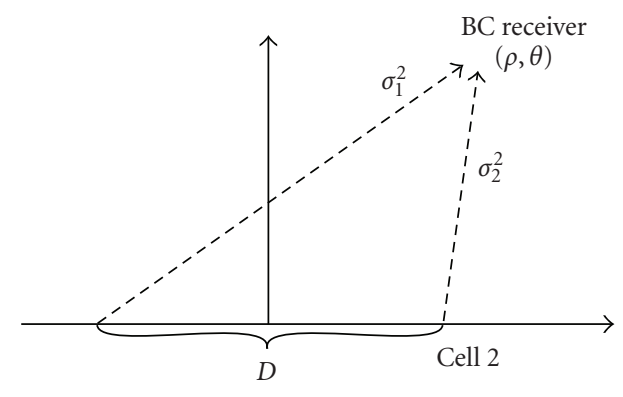

FIgURE 2: Multicell broadcast coverage.

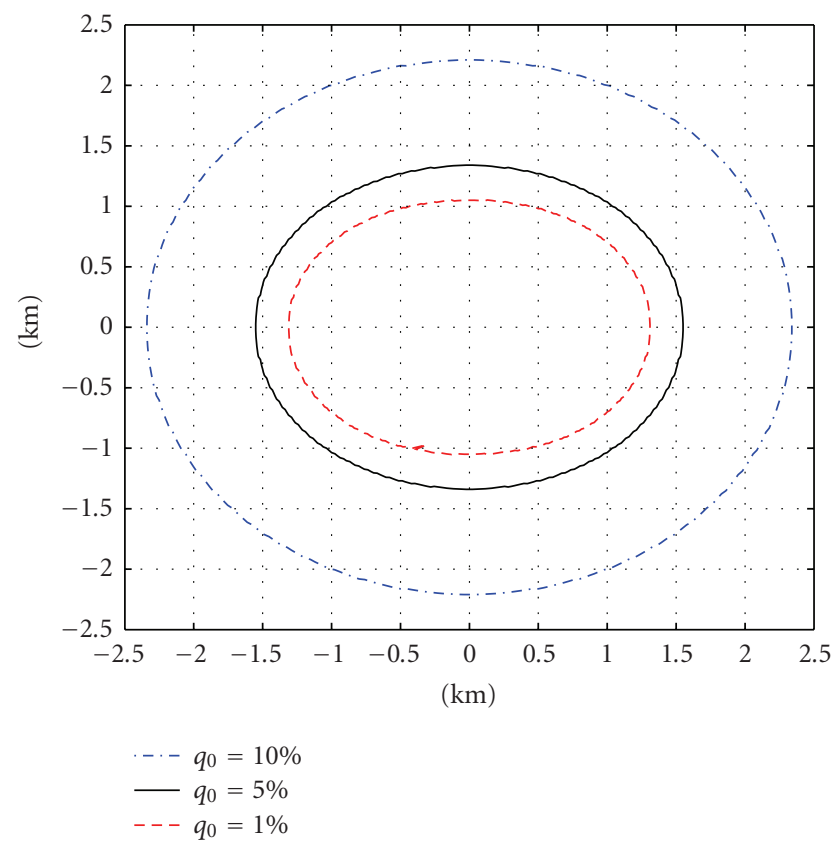

FIgure 3: MISO with antenna location $(0.5,0)$ and $(-0.5,0)$.

Similarly, Figure 4 plots the two cell coverage where the distance between two base stations is doubled. Compared to single cell case, the coverage gains are 3.5245, 2.1469 and 3.5033 for outage probability $1 \%, 5 \%$ and $10 \%$ respectively. We observe that the two cell coverage gain is not sensitive to cell separation distance.

For $N_{t}=3$, Figure 5 shows the broadcast coverage area for both single-cell and multicell transmissions with $q_{o}=5 \%$ [16]. The circle in the centre indicates the single cell coverage area. The outer region is the extended coverage area with multicell cooperation, and the three small circles around base stations are the multicell coverage area when they do not collaborate. Compared to the single cell case, the multicell cooperation coverage gain is $315 \%$ in this case.

To further find the optimal cell separation, we numerically calculate the coverage area as a function of cell separation distance for different $q^{o}$ s. Assume three collaborating cells are equally separated, Figure 6 shows the total coverage area as a function of BS separation distance [16]. The optimal cell separation is $1.8,2.4$ and $2.8 \mathrm{kms}$ to achieve the

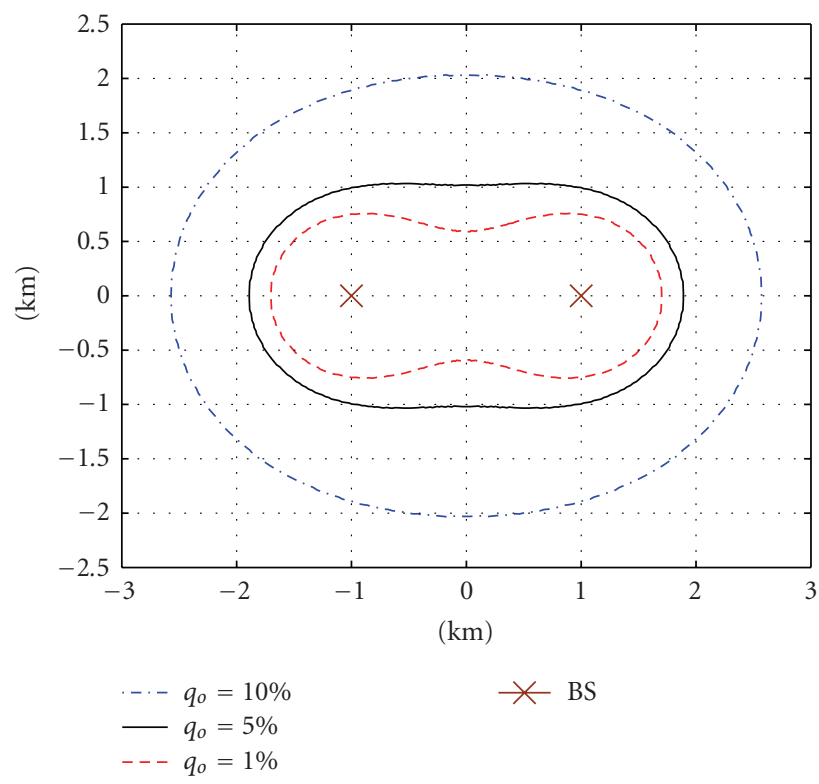

Figure 4: MISO with antenna location $(1,0)$ and $(-1,0)$.

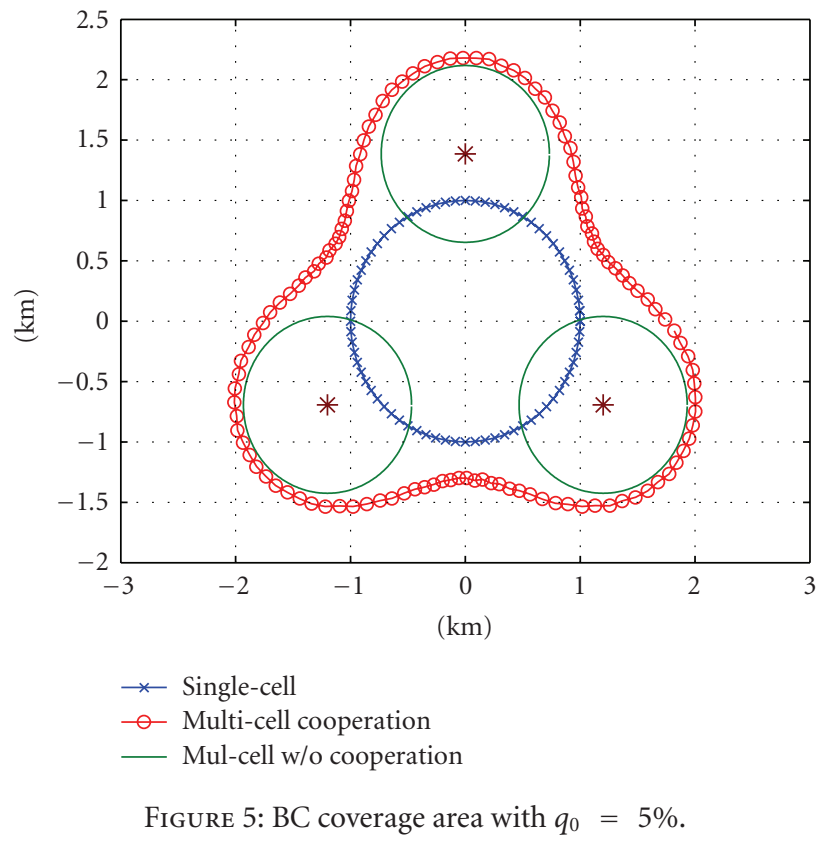

maximum coverage area for outage probability $1 \%, 5 \%$ and $10 \%$, respectively.

3.2. MCC with Multiple Receiver Antenna $\left(N_{r}=N_{t}=2\right)$. When the receiver has multiple antennas, the collaborative multicell broadcast network becomes a distributed MIMO system. While spatial channels are independent among base stations, they are correlated among receiving antennas of each receiver because multiple receiving antennas are closely located. In MIMO broadcast, the outage probability 


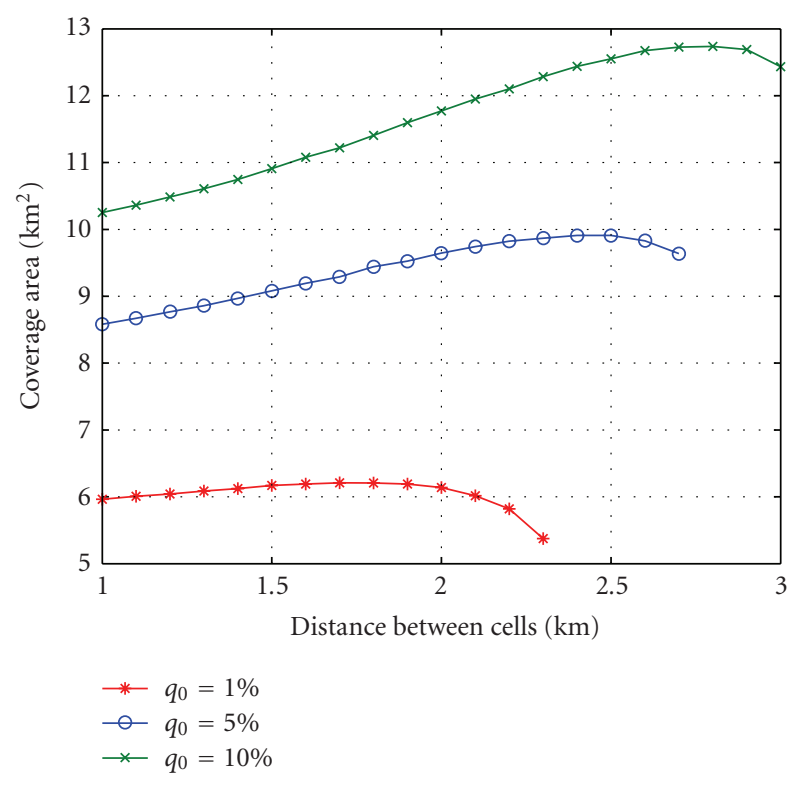

FIGURE 6: BC coverage area versus cell distance $\left(N_{t}=3\right)$.

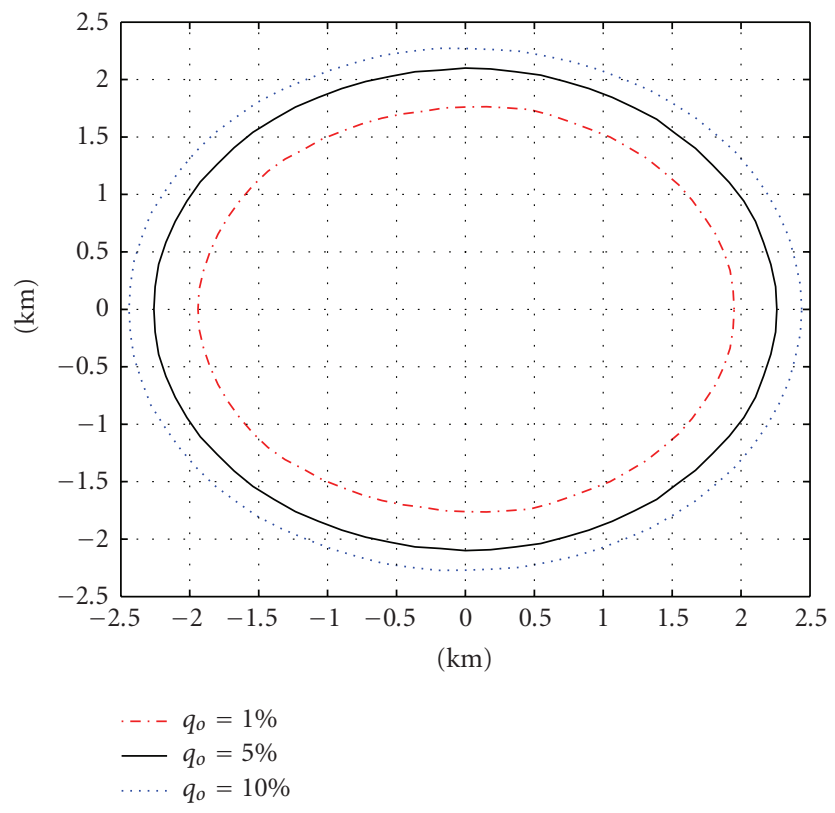

Figure 7: MIMO with antenna location $(0.5,0)$ and $(-0.5,0)$.

associated with receiver $k$ is given by:

$$
\begin{array}{r}
q_{o}=\operatorname{Prob}\left[\mathbf{H}_{k}: B \log _{2} \operatorname{det}\left(\mathbf{I}+\frac{\mathbf{H}_{k} \mathbf{Q} \mathbf{H}_{k}^{H}}{N_{0} B}\right)<r_{o}\right] \\
\text { s.t. } \mathbf{E}\left[x(i) x(i)^{*}\right]=\frac{P}{N_{t}}, \quad 1 \leq i \leq N_{t},
\end{array}
$$

where $\mathbf{H}_{k}$ is the channel matrix associated with user $k$. Plug in $\mathbf{Q}=\left(\mathbf{P} / \mathbf{N}_{\mathbf{t}}\right) \mathbf{I},(12)$ becomes

$$
q_{o}=\operatorname{Prob}\left[\mathbf{H}_{k}: B \log _{2} \operatorname{det}\left(\mathbf{I}+\frac{P \mathbf{H}_{k} \mathbf{H}_{k}^{H}}{N_{t} N_{0} B}\right)<r_{o}\right] .
$$

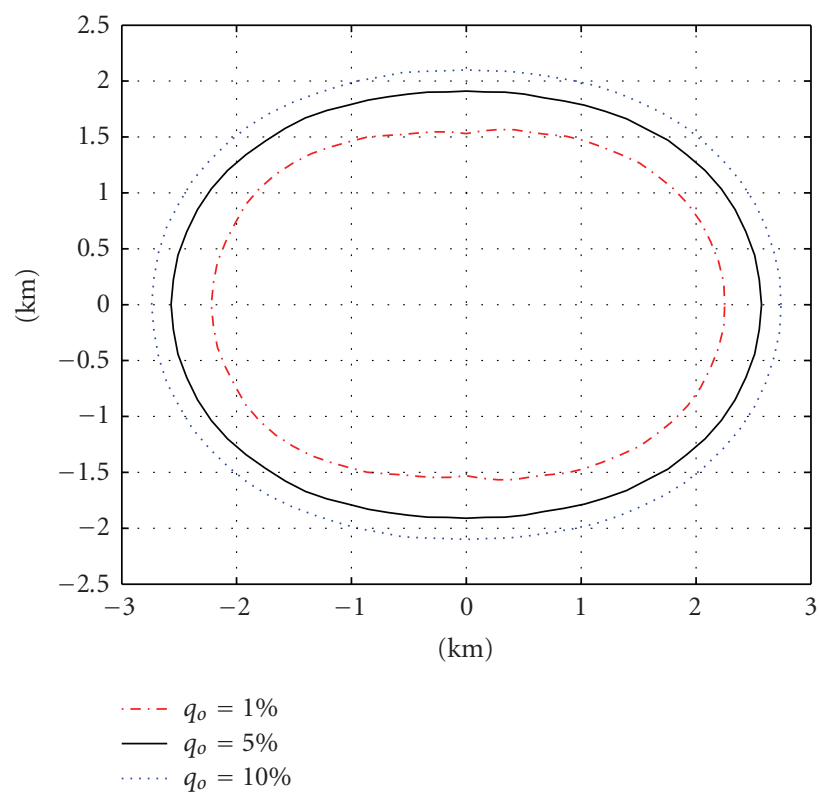

FIgURE 8: MIMO with antenna location $(1,0)$ and $(-1,0)$.

Due to the added spatial complexity, there is no close form for the distribution of $\operatorname{det}\left(\mathbf{I}+P \mathbf{H}_{k} \mathbf{H}_{k}^{H} / N_{t} N_{0} B\right)$ in (13). We thus use simulation to obtain the outage coverage numerically. In our simulation, the spatial channel correlation coefficient between any two receiving antennas is 0.5 .

For the case of $N_{r}=N_{t}=2$, Figure 7 shows the coverage gain with two base stations located at $\left(d_{0} / 2,0\right)$ and $\left(-d_{0} / 2,0\right)$. Compared to SISO broadcast network, the coverage gains are $8.8,4.84$ and 3.71 for outage probability $1 \%, 5 \%$ and $10 \%$ respectively. This huge gain is expected because of the added antenna at each receiver.

Similar results in Figure 8 show the coverage gain with base station locations $\left(d_{0}, 0\right)$ and $\left(-d_{0}, 0\right)$. The coverage gains in this case are $9.21,5.13$ and 3.95 for outage probability $1 \%, 5 \%$ and $10 \%$ respectively. Compared to Figures 7 and 8 yields a better gain due to a larger BS separation. However, the MCC gain will become zero when collaborating BSs are too far away. Therefore, the BSs separation distance should be carefully selected in order to optimize the network coverage. In all cases, we see the distributed MIMO broadcast network provides the maximum coverage.

\section{Conclusion}

In this paper, we studied the performance of multicell cooperation in broadcast network. Due to the unique nature of broadcast, we chose outage capacity and the coverage area as the figure of merit. In reality, cooperation is not feasible among a large number of cells. For the cases of two and three collaborating cells, we explicitly derived the distribution of the received power and calculated the broadcast coverage. We also evaluated the effect of base station separation on broadcast coverage gain. The results show that collaborating BSs should not be placed too close or two far way in order to achieve the maximum coverage. For the distributed 
MIMO case where each receiver has multiple antennas, we provide numerical results to show the expanded coverage area. In all cases, our results show that the coverage area of broadcast network can be significantly increased by multicell cooperation.

\section{References}

[1] S. Shamai (Shitz) and B. M. Zaidel, "Enhancing the cellular downlink capacity via co-processing at the transmitting end," in Proceedings of the 53rd IEEE Vehicular Technology Conference (VTC '01), vol. 3, pp. 1745-1749, Rhodes, Greece, May 2001.

[2] S. Shamai (Shitz), O. Somekh, and B. M. Zaidel, "Multicell communications: an information theoretic perspective," in Proceedings of the Joint Workshop on Communications and Coding (JWCC '04), Florence, Italy, October 2004.

[3] O. Somekh, B. M. Zaidel, and S. Shamai (Shitz), "Sum rate characterization of joint multiple cell-site processing," in Proceedings of the 9th Canadian Workshop on Information Theory (CWIT '05), Montreal, Canada, June 2005.

[4] O. Somekh, O. Simeone, Y. Bar-Ness, A. M. Haimovich, U. Spagnolini, and S. Shamai (Shitz), "An information theoretic view of distributed antenna processing in cellular systems," in Distributed Antenna Systems: Open Architecture for Future Wireless Communications, pp. 31-64, Auerbach, CRC Press, Boca Raton, Fla, USA, 2007.

[5] S. Jing, D. N. C. Tse, J. B. Soriaga, J. Hou, J. E. Smee, and R. Padovani, "Multicell downlink capacity with coordinated processing," EURASIP Journal on Wireless Communications and Networking, vol. 2008, Article ID 586878, 19 pages, 2008.

[6] Y. Kim, H. Li, and H. Liu, "Capacity of a multi-cell cooperative system," in Proceedings of the IEEE International Symposium on Wireless Communication Systems (ISWCS '08), pp. 314-318, Reykjavik, Iceland, October 2008.

[7] O. Simeone, O. Somekh, H. V. Poor, and S. Shamai (Shitz), "Distributed MIMO in multi-cell wireless systems via finitecapacity links," in Proceedings of the 3rd IEEE International Symposium on Communications, Control, and Signal Processing (ISCCSP '08), pp. 203-206, March 2008.

[8] O. Somekh, B. M. Zaidel, and S. Shamai (Shitz), "Spectral efficiency of joint multiple cell-site processors for randomly spread DS-CDMA systems," IEEE Transactions on Information Theory, vol. 53, no. 7, pp. 2625-2637, 2007.

[9] E. Aktas, J. Evans, and S. Hanly, "Distributed decoding in a cellular multiple-access channel," in Proceedings of the IEEE International Symposium on Information Theory (ISIT '04), $\mathrm{p}$. 484, Chicago, Ill, USA, June-July 2004.

[10] A. Sanderovich, O. Somekh, and S. Shamai (Shitz), "Uplink macro diversity with limited backhaul capacity," in Proceedings of the IEEE International Symposium on Information Theory (ISIT '07), pp. 11-15, Nice, France, June 2007.

[11] O. Shental, A. J. Weiss, N. Shental, and Y. Weiss, "Generalized belief propagation receiver for near-optimal detection of twodimensional channels with memory," in Proceedings of the IEEE Information Theory Workshop (ITW '04), pp. 225-229, San Antonio, Tex, USA, October 2004.

[12] P. Marsch and G. Fettweis, "A framework for optimizing the downlink performance of distributed antenna systems under a constrained backhaul," in Proceedings of the 13th European Wireless Conference (EW'07), Paris, France, April 2007.

[13] P. Marsch and G. Fettweis, "A framework for optimizing the uplink performance of distributed antenna systems under a constrained backhaul," in Proceedings of the IEEE International
Conference on Communications (ICC '07), pp. 975-979, Glasgow, Scotland, June 2007.

[14] L. Liu, J. Zhang, J. Yu, and J. Lee, "Inter-cell interference coordination through limited feedback," accepted by International Journal of Digital Multimedia Broadcasting.

[15] M. Eriksson, "Dynamic single frequency networks," IEEE Journal on Selected Areas in Communications, vol. 19, no. 10, pp. 1905-1914, 2001.

[16] H. Li, B. Liu, and H. Liu, "Transmission schemes for multicarrier broadcast and unicast hybrid systems," IEEE Transactions on Wireless Communications, vol. 7, no. 11, pp. 4321-4330, 2008.

[17] A. Goldsmith, Wireless Communications, Cambridge University Press, New York, NY, USA, 2005.

[18] E. Telatar, "Capacity of multi-antenna Gaussian channels," Internal Technical Memorandum, AT\&T Bell Laboratories, June 1995. 

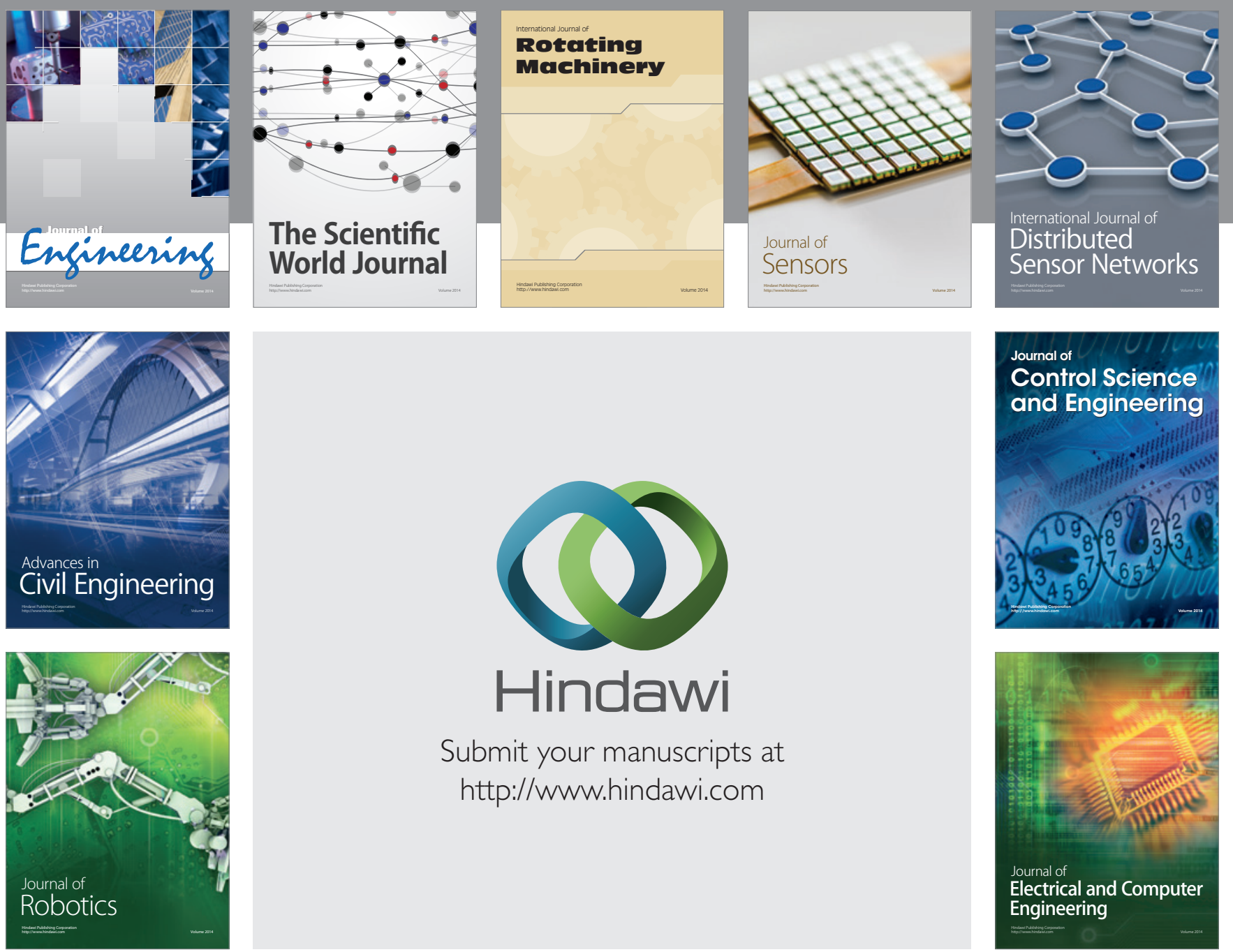

Submit your manuscripts at

http://www.hindawi.com
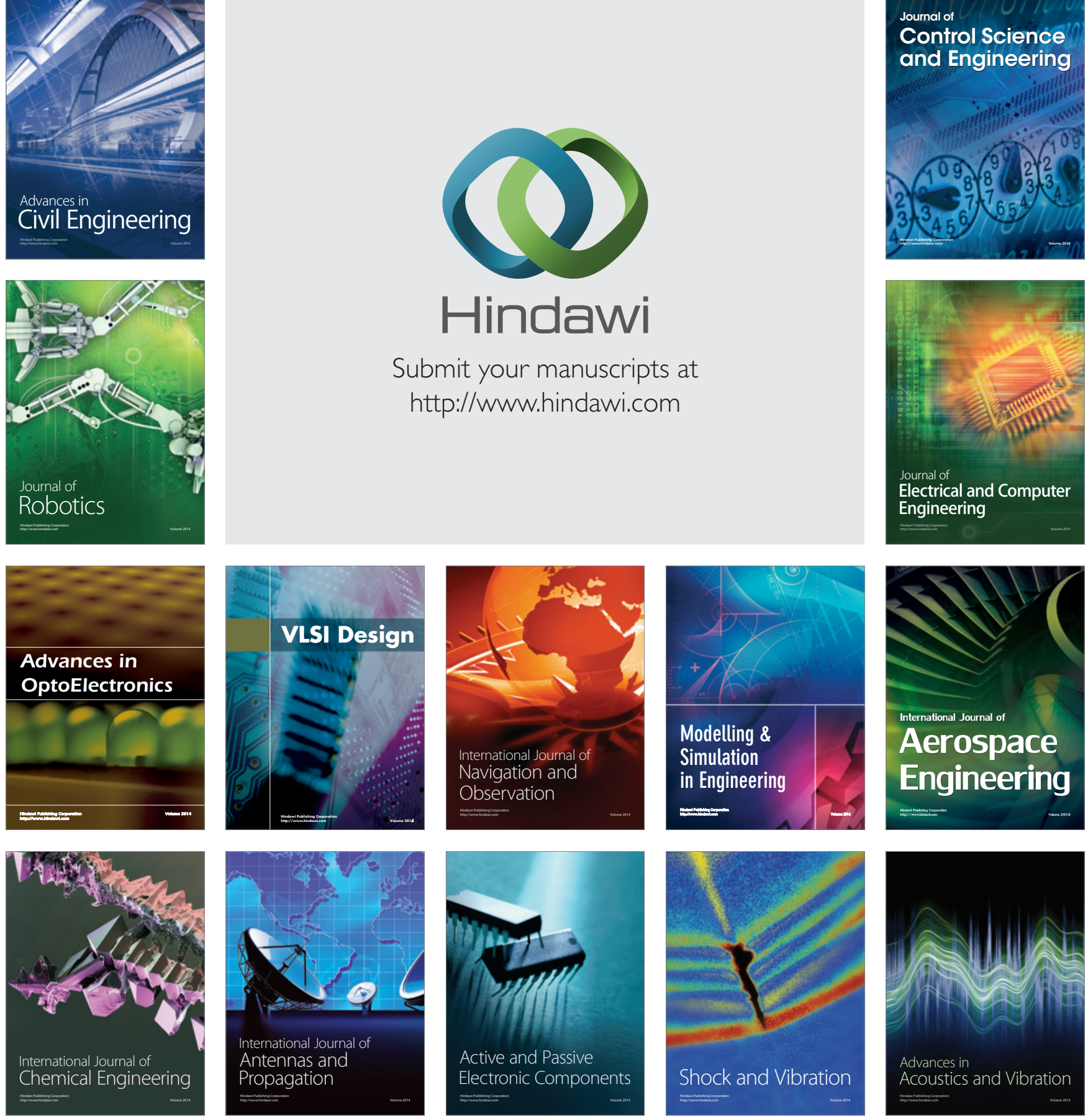